\title{
Border maneuvers: deployment of mucosal immune defenses against Toxoplasma gondii
}

\author{
SB Cohen ${ }^{1}$ and EY Denkers ${ }^{1}$
}

Toxoplasma gondii is a highly prevalent protozoan pathogen that is transmitted through oral ingestion of infectious cysts. As such, mucosal immune defenses in the intestine constitute the first and arguably most important line of resistance against the parasite. The response to infection is now understood to involve complex three-way interactions between Toxoplasma, the mucosal immune system, and the host intestinal microbiota. Productive outcome of these interactions ensures resolution of infection in the intestinal mucosa. Nonsuccessful outcome may result in emergence of proinflammatory damage that can spell death for the host. Here, we discuss new advances in our understanding of the mechanisms underpinning these disparate outcomes, with particular reference to initiators, effectors, and regulators of mucosal immunity stimulated by Toxoplasma in the intestine.

\section{INTRODUCTION}

The intestinal mucosa forms a dynamic arena in which principles of tolerance and immunity are dramatically played out throughout the host lifetime. ${ }^{1-3}$ Effective responses maintain homeostasis and protect from infection; however, dysregulated immunity can lead to inability to control microbial incursion and may lead to chronic inflammatory disease in the gut mucosa and beyond. ${ }^{4-6}$

The structure of the intestine sets the landscape within which these fundamentals of mucosal immunology are played out. The intestinal mucosa is composed of a single layer of epithelial cells with an embedded population of non-conventional intraepithelial lymphocytes. Underlying this, the lamina propria (LP) contains diverse cell types responsible for defense against invading pathogens, including $\mathrm{T}$ cells, macrophages, and dendritic cells (DCs), as well as those that maintain tolerance to commensal flora in the gut. Within the intestinal lumen itself, the vast number of commensals (on the order of $10^{14}$ in humans, with several thousand discrete species normally present) underscores the astounding ability to detect and respond to incoming pathogens while simultaneously avoiding inappropriate reactions to harmless or even beneficial occupants of the intestine. Studies largely in mice with the orally acquired protozoan parasite Toxoplasma gondii have served to illuminate these principles and have contributed key insights into inflammation and immunity in the intestinal mucosa during infection.
Toxoplasma is an exceedingly common parasite of humans and animals, and it is estimated that $10-50 \%$ of the human population are latently infected with the parasite worldwide. The vast majority of cases are asymptomatic, but the parasite may emerge as a life-threatening opportunistic pathogen in immunodeficient hosts and during congenital infection. ${ }^{7}$ Infection is acquired by ingestion of tissue cysts or oocysts shed by cats. Within the small intestine, T. gondii differentiates into the fast replicating and disseminating form known as the tachyzoite. ${ }^{8}$ Invasion of host cells by tachyzoites results in creation of a specialized parasitophorous vacuole within which Toxoplasma replicates, eventually leading to egress and entry into new host cells. Infection leads to spreading beyond the intestinal mucosa and culminates in cyst formation within the skeletal muscle and central nervous system tissues. Within the latter tissue, toxoplasmic encephalitis may emerge as a lifethreatening disease in immunosuppressed patients. In the feline intestine, the parasite undergoes sexual reproduction, resulting in fecal shedding of highly infectious oocysts into the environment. T. gondii is known for its ability to induce a strongly polarized Th1 response that is normally highly effective in host protection but that can also spiral out of control to cause host tissue damage and sometimes death. ${ }^{9-11}$ Although Toxoplasma spreads well beyond the borders of the intestinal mucosa, it is within this tissue that the first encounter between the parasite and host immune system occurs. As such, the

${ }^{1}$ Department of Microbiology and Immunology, College of Veterinary Medicine, Cornell University, Ithaca, New York, USA. Correspondence: EY Denkers (eyd1@cornell.edu) Received 28 January 2014; accepted 13 March 2014; published online 9 April 2014. doi:10.1038/mi.2014.25 
mucosal immune response is the key to determine the outcome of infection with this microbial pathogen.

\section{EARLY MUCOSAL TARGETS OF INFECTION}

In order to establish successful infection, Toxoplasma must breach the intestinal epithelial layer, after which it must disseminate and convert to the life-long latent form. Although tachyzoites may directly invade intestinal epithelial cells, in vitro studies employing polarized intestinal cell lines suggest that the parasites use a more efficient transmigration mechanism dependent on a paracellular pathway. This pathway involves the manipulation of host cell intercellular cell adhesion molecule- 1 and occludin to pass through cellular tight junctions. ${ }^{12,13}$ Interestingly, transmigratory capability seems to be genetically controlled in Toxoplasma and is linked to virulence of the parasite. ${ }^{14,15}$

Once through the epithelial barrier, $T$. gondii invades numerous cell types within the small intestinal LP. Parasites genetically engineered to express fluorescent markers were used to identify infected LP cells after infection, where monocytes and neutrophils were identified as the most frequently infected cells, followed by macrophages and lymphocytes, whereas DCs comprised a low percentage of the infected population. ${ }^{16,17} \mathrm{~T}$. gondii forms discrete foci of replication within the small intestinal villi, and two recent studies provide unexpected evidence that this occurs because the parasite travels in a retrograde manner through the intestinal lumen rather than the tissue to establish localized centers of infection. Interestingly, it also appears to be the case that infected LP neutrophils may transmigrate into the lumen to establish new infection foci. ${ }^{16,17}$ A related role for retrograde neutrophil transmigration in response to Toxoplasma comes from a recent study showing that neutrophils emigrate into the intestinal lumen shortly after infection to form structures that encapsulate commensals, limiting their entry into the intestinal mucosa that would otherwise occur. ${ }^{18}$

DCs are well known for their capacity to take up soluble or particulate antigen and migrate to regional lymphoid organs. As such, they have also been proposed to be "Trojan horses" for parasite dissemination during oral and intraperitoneal infection. ${ }^{19,20}$ Interestingly, DCs infected with $T$. gondii acquire a hypermotility phenotype that is dependent upon parasite exploitation of GABAergic signaling in the host cell. ${ }^{21,22}$ Adoptive transfer of infected DC results in faster parasite dissemination and increased parasite burden compared with the transfer of free parasites. ${ }^{23}$ However, it is yet to be established that this phenomenon also operates in the intestinal mucosa. Regardless, whereas some studies indicate that LP DCs are not a major infection reservoir, they may nevertheless contribute to dissemination of parasites from the mucosa into regional lymph nodes and beyond.

\section{ROLE OF MUCOSAL DCS IN INNATE IMMUNE INITIATION}

Concomitant with early invasion in the intestinal mucosa, it is essential for host resistance that interleukin (IL)-12 production is initiated to ensure the production of protective interferon (IFN)- $\gamma$ by natural killer (NK) cells and newly generated Th1 T lymphocytes. Several studies have characterized cellular sources of IL-12 following oral $T$. gondii infection. Although in vitro studies identify neutrophils, DCs, and inflammatory monocytes as cells that produce IL-12 in response to T. gondii, the relative role of these cells during in vivo infection is less clear. ${ }^{24-27}$ While polymorphonuclear leukocyte may be an important in vivo source of IL-12 stimulating protective immunity, more current data indicate that neutrophil recruitment to the intestine contributes to small intestinal inflammatory pathology that is triggered by Toxoplasma. ${ }^{28,29}$

DCs are implicated as a primary source of IL-12 based upon intraperitoneal (IP) and oral infection studies using the Cre-flox and diphtheria toxin systems, respectively, to delete cells expressing CD11c, a marker associated with DC. ${ }^{24,30}$ In the case of oral infection, ablation of $\mathrm{CD} 11 \mathrm{c}^{+}$cells leads to elevated parasite burden and loss of the $\mathrm{CD} 4^{+}{ }^{+}$-cell IFN- $\gamma$ response. An important caveat in these studies is that CD11c can also be expressed by inflammatory monocytes, which themselves are observed to produce IL-12 during infection. ${ }^{28,29,31}$ Nevertheless, mice lacking Batf3, a transcription factor important for differentiation of CD $8 \alpha$ and tissue CD103 DC, ${ }^{32,33}$ are defective in IL-12 production during Toxoplasma infection, providing compelling evidence for these specific DC subsets as sources of IL-12 during oral infection. ${ }^{34}$ Among these, CD103 DC may be the prime IL-12 source in the intestinal mucosa, as a previous study showed that intestinal $\mathrm{CD} 8 \alpha^{-}$, but not $\mathrm{CD} 8 \alpha^{+} \mathrm{DC}$, express IL-12 following oral infection. ${ }^{35}$

A recent study employed diphtheria toxin to ablate cells expressing a newly identified transcription factor zDC (Zbtb46, Btbd4), which is exclusively expressed by conventional DC subsets and not by monocytes or plasmacytoid DC. ${ }^{30}$ Mice that were ablated for these cells and then orally infected with T. gondii failed to reach the same level of susceptibility and reduced Th1 activity as CD11c-depleted mice. This suggests that non-conventional DC or other cells that express CD11c, possibly including inflammatory monocytes, may also contribute to the establishment of protective immunity to T. gondii. ${ }^{30}$ One scenario that seems particularly likely is that resident $\mathrm{CD}_{103}{ }^{+}$DCs in the intestine provide the initial burst of IL-12, and this is followed by activation and recruitment of inflammatory monocytes to the intestinal mucosa that might further contribute to the IL-12 response during infection, enabling optimal Th1 generation.

In addition to producing IL-12, mucosal DCs have been shown to regulate $\mathrm{T}$-cell responses following $T$. gondii infection by production of the vitamin A metabolite retinoic acid (RA). Production of RA by mucosal DC promotes tolerogenic responses at steady state by acting in concert with transforming growth factor beta to induce mucosal regulatory $\mathrm{T}$ cell (Treg). ${ }^{36-38}$ As RA directly binds to T cells via RA receptor- $\alpha$ and enhances $\mathrm{T}$-cell receptor signaling, ${ }^{39}$ more generalized effects of RA on T cells are possible. Indeed, loss of RA receptor$\alpha$ signaling in the context of inflammation initiated by Toxoplasma infection results in defective T-cell effector responses in the mucosal compartment and increased susceptibility to the parasite. ${ }^{39}$ 


\section{IMMUNE RECOGNITION}

How T. gondii is detected by the immune system and the role of Toll-like receptors (TLRs) in this lynchpin event has been the subject of intense interest and investigation. ${ }^{40-42}$ Mice deficient in MyD88 and IRAK4, the central mediators of TLR signaling, are extremely susceptible to Toxoplasma infection. ${ }^{43-45}$ Furthermore, deficiency in UNC93B, a chaperone protein involved in trafficking intracellular TLR from the endoplasmic reticulum to the endosomal compartment, results in complete loss of resistance to $T$. gondii. ${ }^{46,47}$ These results together point to intracellular TLR as being particularly important in immune detection of Toxoplasma.

The tachyzoite protein profilin (TgPRF) serves as an IL-12inducing ligand for TLR11, which along with TLR3, 7, 8, 9, and 12 , is expressed intracellularly. ${ }^{47,48}$ More recently, it was found that TgPRF also activates immunity through binding to TLR12. ${ }^{49-51}$ Current evidence indicates that TgPRF recognition by TLR11 or TLR12 homodimers or TLR11/12 heterodimers is a major event in innate immune recognition of Toxoplasma. Which particular TLR configuration takes primary importance may depend upon the cell type involved or the context of infection. Whereas this is an area that is yet to be explored in detail, results in $T h 11^{-/}$mice indicate that signaling mediated by this receptor plays a role in proinflammatory Crohn's disease-like pathology in the intestine that is triggered by high-dose infection. ${ }^{35}$

Tachyzoite profilin is a molecule that is required for cell invasion. ${ }^{52}$ As a molecule essential for parasite survival, it fulfills a basic requirement for a pathogen-associated molecular pattern targeted for recognition by the immune system. ${ }^{53}$ The TgPRF protein is not actively secreted by Toxoplasma, and TLR11/12 do not observably traffic to the parasitophorous vacuole membrane. Therefore, it is most likely that recognition at this level involves DC phagocytic uptake of dead parasites or debris, and indeed there is evidence that most IL-12 is produced by noninfected cells. ${ }^{54}$

In addition to recognition of TgPRF by intracellular TLR, parasite nucleic acid recognition by TLR3, 7, and 9 also facilitates resistance to infection. ${ }^{49}$ Furthermore, TLR2 and TLR4 recognition of parasite glycosylphosphatidylinositol lipids at the cell surface may contribute to innate immune activation, although conclusions from these studies rely mostly on in vitro observations. ${ }^{55,56}$ Regardless, the sum of these data strongly argues that several TLR, most prominently TLR11 and TLR12, act in concert to signal recognition of $T$. gondii. However, TLR11 and TLR12, while being expressed in rodents, are absent in many species, including humans. In such cases, TgPRF is most likely less important, and other parasite molecules are likely to take the role of primary innate immunity triggers. It is possible that parasite nucleic acids and glycosylphosphatidylinositol lipids emerge as dominant TLR ligands in these cases.

The secretory dense granule protein GRA15, a molecule expressed in a parasite strain-dependent manner by low virulence Toxoplasma, has been shown to trigger NFKB activation and subsequent IL-12 production directly within infected cells. ${ }^{57}$ This is at odds with data indicating that Toxoplasma is recognized at a distance by noninfected cells; however, some studies do in fact indicate that IL-12 is produced by infected cells. ${ }^{58}$ One recently proposed model that resolves the apparent discrepancy is that IL-12 at earliest points of infection may be produced by infected cells through GRA15. Later with the onset of fulminant infection, TgPRF uptake and TLR stimulation in noninfected cells provides the major source of IL-12. ${ }^{59}$ Nevertheless, the strain specificity of GRA15 expression would indicate that the cellular origins of IL-12 production must depend upon the infecting Toxoplasma isolate.

\section{LUMINAL BACTERIA AND TOXOPLASMA TOGETHER CONTROL MUCOSAL IMMUNITY DURING INFECTION}

Our understanding of innate immune recognition of Toxoplasma has outpaced similar knowledge of other eukaryotic microorganisms. Nevertheless, it has become clear that our understanding remains incomplete insofar as simultaneous recognition of luminal gut flora must also be considered. Commensal bacteria benefit the host by shaping mucosal immunity under steady-state conditions; however, they also cause inflammation under disequilibrium situations. In the context of Toxoplasma in the intestine, the host microbiota has similar roles.

Data pointing to the importance of the microbiota in oral T. gondii infection come from several lines of evidence. Following IP infection, TLR11 deficiency results in complete loss of DC-derived IL-12 and the subsequent IFN- $\gamma$ response upon systemic T. gondii infection, and mice fail to control the infection, resulting in early mortality. However, these responses, although diminished, are nevertheless retained in the intestinal mucosa of $T l r 11^{-/-}$mice during mucosal infection, which is in direct contrast to infection in $M y D 88^{-/-}$ animals. Furthermore, antibiotic treatment of $\operatorname{Tr} 11^{-/-}$ animals before T. gondii infection abolishes the remaining IL-12 and Th1 responses and increases susceptibility to oral infection, demonstrating the importance of gut bacteria in providing an immunostimulatory role in the absence of TgPRF recognition. ${ }^{35}$ Thus, the gut commensal population acts as an adjuvant facilitating development of an optimal protective immune response to Toxoplasma.

It was recently shown that oral infection with $T$. gondii directs an antigen-specific immune response not only against the parasite but also against the microbiota itself. Through the use of mice genetically engineered to express a T-cell receptor specific for commensal-derived flagellin, Hand et al. ${ }^{60}$ showed that a population of $\mathrm{CD}^{+} \mathrm{T}$ cells induced by $T$. gondii infection responds directly to bacterial flagellin through proliferation and Th1 differentiation. Surprisingly, the commensal-specific T-cell population is comparable to that of the parasite-specific cells both in size and behavior. Furthermore, these flagellin-specific cells develop into functional memory cells. T. gondii infection in the gut, therefore, seems to trigger loss of tolerance to intestinal bacteria, possibly due to infectioninduced epithelial damage and consequent bacterial translocation. In turn, this results in $\mathrm{T}$-cell priming against bacterial 
antigens; however, the bacteria also provide a source of PAMPs that facilitates ignition of the protective Th1 response against Toxoplasma itself (Figure 1).

It is also clear that gut bacteria can exacerbate $T$. gondii infection in a manner that is similar to that thought to occur in inflammatory bowel disease pathogenesis. ${ }^{5}$ High-dose oral infection with $T$. gondii leads to an inflammatory bowel diseaselike phenotype in C57BL/6 mice, which is associated with $\mathrm{CD} 4{ }^{+} \mathrm{T}$-cell infiltration and overexpression of proinflammatory cytokines, including IFN- $\gamma$, IL-12, and TNF- $\alpha$, as well as IL-22 and possibly IL-17 (although this is controversial). ${ }^{61-65}$ Additionally, T. gondii alters the composition of the gut microbiota, resulting in a global reduction in bacterial diversity, a switch from Gram-positive to Gram-negative bacteria, and an expansion of adherent and invasive pathobionts that results in translocation into the LP. ${ }^{66,67}$

It is clear that imbalances in the intestinal microbiota are a cause of inflammation during T. gondii infection. For example, administration of antibiotics before Toxoplasma infection protects mice from parasite-induced ileitis, and gnotobiotic mice are resistant to parasite-induced ileitis, confirming the role of the microbiota in promoting intestinal lesions upon T. gondii infection. ${ }^{67}$ Mice deficient for TLR4 display decreased

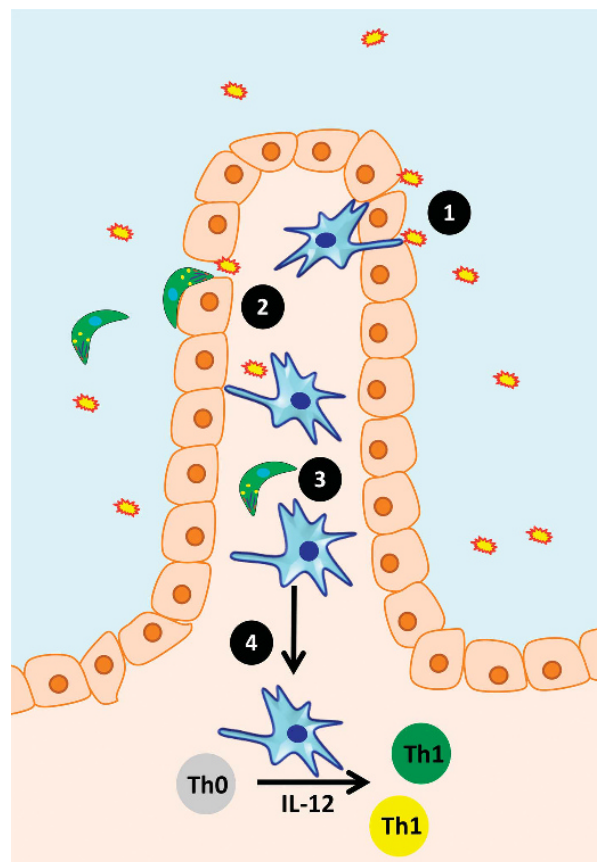

Figure 1 Gut commensal bacteria serve as adjuvants and antigens for Toxoplasma-triggered interleukin (IL)-12 and Th1 induction. (1) Gutresident CX3CR $1^{+}$macrophages or dendritic cells (DCs) continually sample bacterial antigens from the intestinal lumen by extending dendrites between epithelial cells. (2) Mucosal DCs may also sample bacteria that have translocated to the LP as a result of Toxoplasma-mediated disruption of the epithelial barrier associated with infection. This may occur during parasite paracellular migration or by direct infection and lysis of epithelial cells. (3) DCs acquire Toxoplasma antigen either by direct infection or by uptake of parasite material. (4) Activated antigen-bearing DCs migrate to the draining lymph node and produce IL-12, resulting in the activation of Th1 cell populations specific for commensals and $T$. gondii itself.
IFN- $\gamma$ and NO levels in the small intestine and are more resistant to infection, most likely resulting from lost recognition of bacterial TLR4 ligands. ${ }^{68}$ Furthermore, antibiotic treatment of recipient mice protects against ileitis induced by adoptive transfer of pathogenic LP CD4 ${ }^{+} \mathrm{T}$ cells and intraepithelial lymphocytes (IELs) from Toxoplasma-infected animals. ${ }^{69}$ These collective studies emphasize the dual potential role of luminal microbiota in stimulating protective immunity with low-dose parasite inocula versus switching to driving an inflammatory cytokine storm that surrounds high-dose infection.

The precise trigger provided by $T$. gondii that results in loss of tolerance to gut flora and intestinal dysbiosis has remained enigmatic; however, recent studies suggest that parasite effects on Paneth cells may be the key to pathogenesis. Paneth cells are specialized epithelial cells located in intestinal crypts, and they secrete antimicrobial peptides such as defensins into the intestinal lumen. ${ }^{70}$ One study found that, early after oral infection, Paneth cells degranulate in a TLR9-dependent manner, resulting in the release of antimicrobial Criptidins that somehow promote optimal Th1 responses to the parasite. ${ }^{71}$ More directly related to the onset of inflammation, it was recently found that Toxoplasma infection causes loss of Paneth cells, an event that in turn leads to dysbiosis and fulminant pathology. ${ }^{72}$ Interestingly, germ-free mice infected with T. gondii retain Paneth cells, although they are again eliminated upon the addition of Enterobacteriaceae, but not Bacteroides. This suggests that Paneth cell loss is dependent on the combined effect of the parasite and Protoeobacteria that emerge as dominant species during intestinal inflammation. Loss of Paneth cells is dependent on CD4 ${ }^{+}$T-cell IFN- $\gamma$ production via T-cell-intrinsic MyD88 signaling, as Lck-Cre $M y d 88^{f l / f l}$ mice phenocopy $M y d 88^{-1-}$ animals, which display unaltered Paneth cells and antimicrobial peptide expression. ${ }^{72}$ Thus, this study provides a model to understand how Toxoplasma triggers inflammation-mediated dysbiosis in the intestine. Early IL-12-dependent induction of Th1 cells specific for Toxoplasma and intestinal flora causes IFN- $\gamma$-dependent Paneth cell depletion. In turn, loss of a major source of antimicrobial peptides in the small intestine results in overgrowth of pathobionts associated with dysbiosis and associated inflammatory lesions (Figure 2). This has particular relevance for understanding ileal Crohn's pathogenesis because independent studies have implicated dysfunctional Paneth cell responses as underlying onset of disease. ${ }^{73}$

\section{ROLE OF INFLAMMATORY MONOCYTES IN THE INTESTINAL MUCOSA}

Whereas inflammatory monocytes may be a significant IL-12 source during $T$. gondii infection, their more important function is most likely as chemokine receptor CCR2-dependent microbicidal effector cells. ${ }^{74}$ Pioneering studies demonstrated the importance of similar cells in defense against Listeria monocytogenes in the spleen. ${ }^{75}$ Subsequently, CCR2-dependent recruitment of these cells into the intestinal mucosa of Toxoplasma-infected mice was found to control parasite 


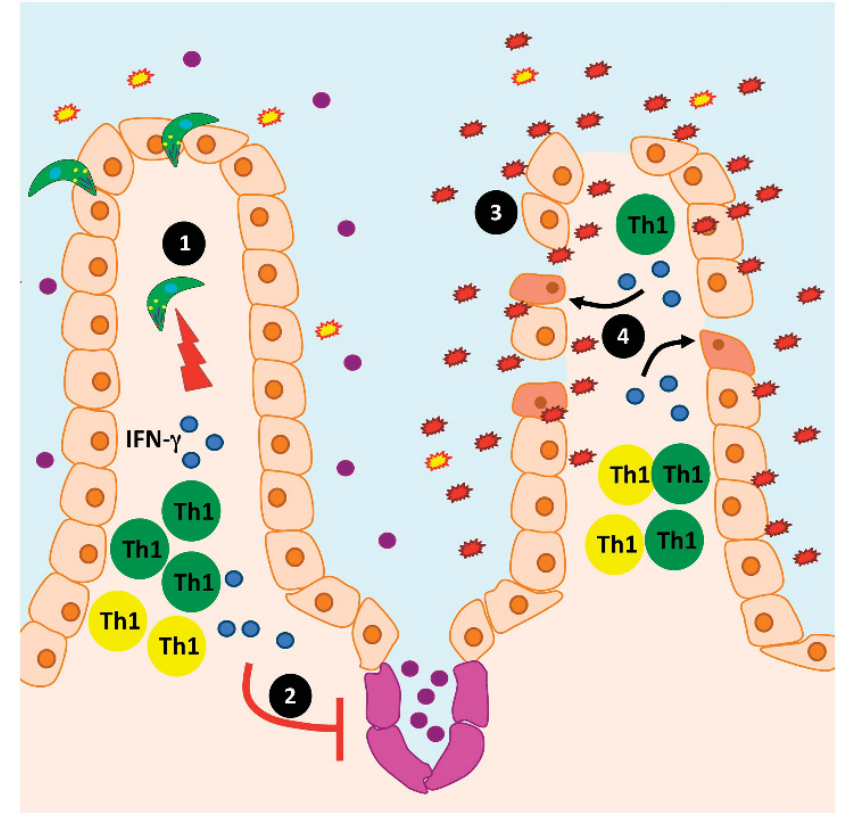

Figure 2 Control of infection and emergence of dysbiosis during Toxoplasmainfection. (1) interferon (IFN)- $\gamma$ produced by Th1 lymphocytes and natural killer (NK) cells (not shown) induces killing of parasites and resolution of infection. The degree to which parasite- and commensalspecific T cells (denoted in yellow and green) contribute to killing is presently unclear. (2) During emergence of dysbiosis, infection-induced IFN- $\gamma$ promotes Paneth cell elimination. Normally, these cells secrete antimicrobial peptides (AMPs) into the intestinal lumen to maintain homeostatic levels of commensal populations, including the healthy Gram-positive Bacteroides (shown in yellow). (3) Expansion of pathogenic Gram-negative Enterobacteriaceae (shown in red), which adhere to the epithelium, cause barrier damage, and invade the intestinal mucosa, is caused by loss of Paneth cell-dependent AMP. (4) Damage may be further exacerbated by the continued proinflammatory activity of Th1 effectors responding to bacteria and parasite antigen.

replication. ${ }^{31}$ Rather than controlling migration to the inflamed intestinal LP, CCR2 appears to be necessary for exit from the bone marrow. ${ }^{76}$ The recruitment of inflammatory monocytes into the intestine now appears to be mediated by a CCL3/CCR1 axis, ${ }^{77}$ a finding that confirms older studies showing a requirement for CCR1 in control of infection. ${ }^{78}$ Thus, LP IL-15-dependent NKp $46^{+}$innate lymphoid cells activated by infection-induced IL-18 produce CCL3 that in turn chemoattracts CCR1-positive inflammatory monocytes to the intestinal mucosa (Figure 3).

Inflammatory monocytes must be activated by IFN- $\gamma$ to acquire effector function. In an IP infection model, NK cells may provide this critical source; ${ }^{79}$ however, our recent studies further identified Th1 $\mathrm{T}$ cells, recruited to the site of infection in dependence upon expression of CXCR3, as an important source of this cytokine in this capacity. ${ }^{28}$ Thus, we propose an intricate chemokine network surrounding Toxoplasma in the intestine, in which CCR1/CCL3 recruits CCR2-dependent monocytes, whereas, approximately simultaneously, Th1 effector cells are recruited through CXCR3 to affect optimal inflammatory monocyte activation for parasite control (Figure 3).
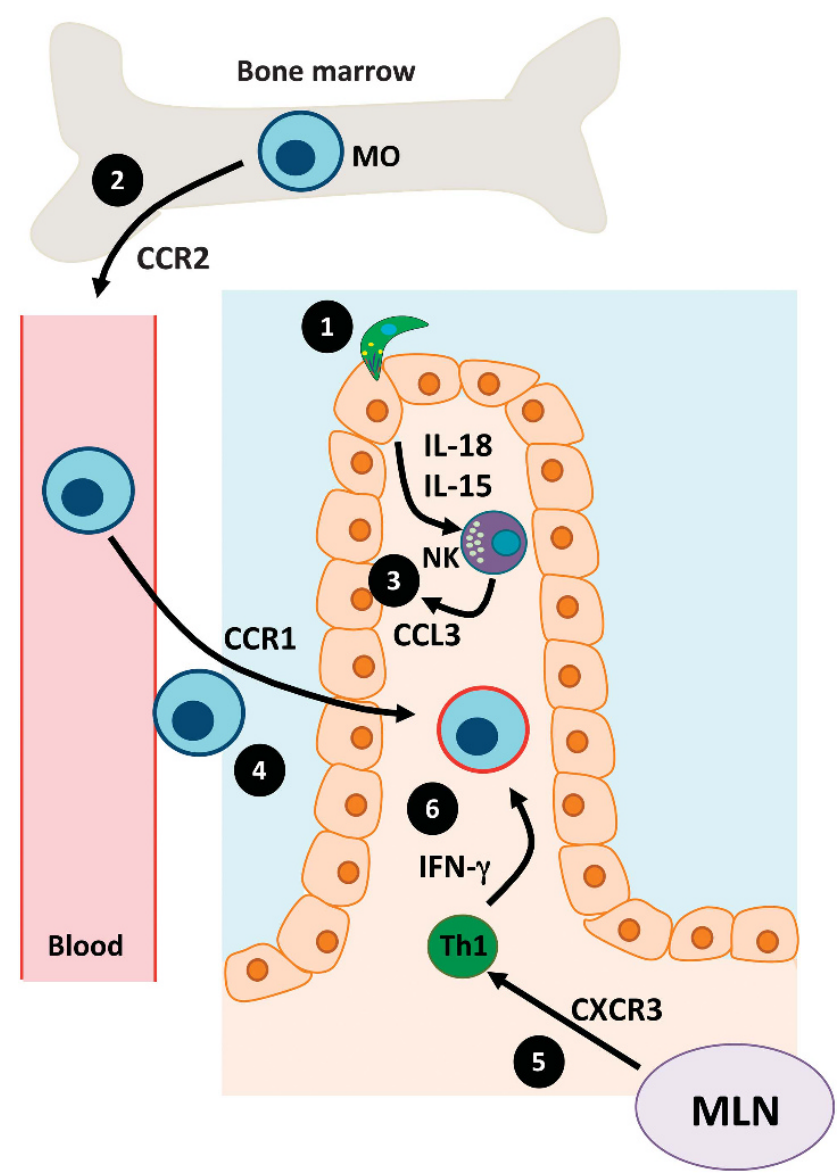

Figure 3 Chemokine network surrounding inflammatory monocyte recruitment and activation during $T$. gondii infection. (1) Upon intestinal infection, non-hematopoietic cells, such as epithelial cells, are induced to secrete cytokines interleukin (IL)-18 and IL-15, resulting in activation and maturation of NKp46 + innate-like lymphocytes. (2) Concurrently, monocytes are recruited from the bone marrow to the bloodstream in dependence upon CCR2. (3) In the intestinal LP, activated, innate-like lymphocytes secrete chemokines, such as CCL3. (4) Recruitment of monocytes expressing CCR1, a receptor for CCL3, promotes accumulation in the intestinal LP. (5) Local production of interferon (IFN)$\gamma$-inducible chemokines following $T$. gondii infection recruits CXCR3expressing Th1 cells presumably from the mesenteric lymph node (MLN). (6) High-level secretion of IFN- $\gamma$ by CXCR3-positive CD4 T cells activates LP inflammatory monocytes, resulting in upregulated antimicrobicidal effector function and enabling control of the parasite.

It is not yet clear how inflammatory monocytes within the intestine kill parasites. The cells were first defined based, in part, on their ability to express inducible nitric oxide synthase and produce the microbicidal molecule nitric oxide. ${ }^{74,80}$ However, because inducible nitric oxide synthase-deficient mice survive acute infection (in contrast to If $n \gamma^{-/-}$mice), ${ }^{81,82}$ this killing mechanism is unlikely. Instead, it is more likely that immunityrelated GTPase (IRG) proteins are the key to parasite killing. IRG effector molecules are strongly induced by IFN- $\gamma$, and recent data show that they accumulate on the parasitophorous vacuole membrane to mediate its destruction, thereby depriving the parasite of its essential intracellular niche. ${ }^{83-86}$ Nevertheless, IRG studies have mostly employed in vitro 
infection systems, and while some IRG knockout mice are indeed increased in susceptibility during oral infection, ${ }^{87}$ formal data demonstrating a requirement for this activity in mucosal inflammatory monocyte killing are lacking. There is also emerging evidence that members of the p65 guanylatebinding protein family have a role in parasite killing, although their contribution to killing in the intestinal mucosa has not yet been determined. ${ }^{88}$

A more complex view of inflammatory monocytes comes from a recent study indicating immunoregulatory properties in response to Toxoplasma. ${ }^{89}$ Thus, these cells acquire a tissuespecific regulatory phenotype that is dependent upon commensals in the gut. This property is associated with production of the lipid mediator prostaglandin E2, a molecule known to have immunoregulatory functions in the intestinal mucosa. ${ }^{90}$ In turn, prostaglandin E2 inhibits activation of neutrophils that also accumulate in the intestinal LP during T. gondii infection. In the absence of CCR2-dependent inflammatory monocyte recruitment, neutrophil activation and concomitant release of factors, such as TNF- $\alpha$ and reactive oxygen species, result in emergence of intestinal lesions. Thus, inflammatory monocytes recruited to the intestine possess the dual properties of controlling parasite growth and downmodulating polymorphonuclear leukocyte-mediated intestinal pathology.

\section{INTRAEPITHELIAL LYMPHOCYTE FUNCTION DURING TOXOPLASMA INFECTION}

IEL constitute a complex population of T cells embedded within the intestinal epithelium. They are normally present at approximately one per 10 epithelial cells, although this can vary considerably depending upon inflammation and infection. In mice, the majority of IELs express either CD $8 \alpha \beta$ or CD $8 \alpha \alpha$ and, among the latter, a large proportion expresses the $\gamma \delta$ rather than $\alpha \beta$ T-cell receptor. The origin, antigen specificity, major histocompatibility complex restriction, and function of these cells are complex and not well understood. ${ }^{91}$ Nevertheless, growing evidence suggests that under normal conditions these cells serve as important regulators of homeostasis, and dysregulation in the IEL compartment contributes to pathology associated with human inflammatory bowel disease. ${ }^{92}$

During T. gondii infection, intestinal epithelial tight junction proteins are abnormally affected and barrier function is compromised, resulting in decreased control of parasite spread. ${ }^{93}$ Others have shown that CD $8 \alpha \beta$ IEL isolated from T. gondii-infected mice produce IFN- $\gamma$ and are capable of cytolytic function against infected enterocytes in vitro. ${ }^{94}$ When adoptively transferred, these IELs traffic to the small intestinal mucosa in dependence upon CCR5 and provide protection against challenge infection. ${ }^{95-97}$ Perhaps most interestingly, IFN- $\gamma$ is required in recipient animals but not donor IEL themselves. $^{98}$

Populations within the IEL compartment also functionally interact with $\mathrm{CD} 4{ }^{+} \mathrm{T}$ cells in the LP. For example, CD $4^{+} \mathrm{T}$ lymphocytes somehow interact with infected epithelial cells to promote proinflammatory cytokine production. ${ }^{99} \mathrm{CD} 8 \alpha \beta$ IELs downregulate this activity in dependence upon transforming growth factor beta production, suggesting that they have a role in protecting against inflammation in the intestine. ${ }^{95,100}$ Nevertheless, within the IEL population as a whole is embedded the potential to contribute to inflammatory pathology during infection. ${ }^{11}$ During high-dose infection in C57BL/6 mice, $\alpha \beta$ T-cell receptor CD8 $\alpha$ IELs mediate damage that is dependent upon their expression of CCR2. ${ }^{101}$ The pathogenic IEL population that emerges under inflammatory conditions does not function alone, but instead requires LP-derived CD4 T cells for fulminant pathology. ${ }^{69}$ In this situation, IELs have a role in recruiting $\mathrm{CD}_{4}^{+} \mathrm{T}$ lymphocytes into the intraepithelial compartment, providing a mechanistic scenario for the original observation that $\mathrm{CD} 4{ }^{+} \mathrm{T}$ cells are required for parasitemediated ileal damage. ${ }^{102}$ Taken together, these data indicate that under low-dose inocula, IELs seem to be important immunoregulatory cells that prevent the onset of intestinal pathology and may even contribute to control of infection. However, under high-dose conditions, these cells switch to a dysregulated proinflammatory phenotype that contributes to lesion development in the small intestine. Whether this reflects actual switching in the function of a single IEL population or instead results from the changing dominance of one IEL population over another is not at present understood. Furthermore, while the gut microbiota is required for the pathogenic effects of IEL, little is known about how the intestinal flora interact and drive the function of these cells.

\section{TOXOPLASMA CONTROLS THE FATE OF INTESTINAL TREG}

Within the intestinal mucosa resides a large population of FoxP3 ${ }^{+}$Tregs composed of steady-state cells (nTregs) and cells induced by infection or inflammation. Recent data suggest that Treg induced by infection express T-bet and depend upon IFN- $\gamma$ in the periphery and IL-27 at mucosal sites. ${ }^{103}$ Tregs are well known to be important in quelling inflammation and maintaining tolerance in the intestine, in large part, through the production of anti-inflammatory IL-10. ${ }^{104}$ During Toxoplasma infection, IL-10 clearly has an important role as $I l-10^{-/-}$mice rapidly succumb due to proinflammatory cytokine overproduction. ${ }^{105}$ Within the context of oral infection, susceptibility due to IL-10 loss is associated with emergence of Crohn's like pathology in the small intestine. ${ }^{106}$

During lethal oral infection, Foxp $3^{+}$Tregs in the intestine express IL-10 and they co-express IFN- $\gamma$ and T-bet. Nevertheless, the intestinal Treg population collapses in size with the emergence of inflammatory pathology. ${ }^{107}$ This was shown to be due to expansion of pathogen-specific $\mathrm{T}$ cells with limited IL-2-producing capacity, leading to the cytokine starvation of Treg. ${ }^{108}$ Thus, reversal of this collapse was accomplished by the addition of recombinant IL-2/anti-IL-2 complexes, resulting in fewer effector T-cell populations and ameliorated pathology. Importantly, the collapse in the Treg population is not restricted to a lethal Toxoplasma dose, as a similar loss in Treg occurs under low-dose non-lethal parasite infection (our unpublished observations and Benson et al. ${ }^{108}$ ). Indeed, it is tempting to speculate that emergence of microbiota-specific $\mathrm{T}$ cells associated with Toxoplasma 
infection is enabled by extinguishing tolerogenic Treg in the intestine.

If Treg disappearance in the intestine is a characteristic of Toxoplasma infection, what is the source of IL-10 that protects against immune-mediated disease? First, it is possible that sufficient numbers of Treg are maintained during nonlethal infection to provide protective IL-10. Second, it is possible that other cell types provide IL-10. The finding that CD4 ${ }^{+}$T-cellderived IL-10 is necessary to prevent immunopathology during oral T. gondii infection does not distinguish between conventional CD4 ${ }^{+}$T lymphocytes and Treg cells. ${ }^{109}$ However, in an IP infection model it was indeed found that IFN- $\gamma$-producing Th1 cells switch to IL-10 production as infection progresses. ${ }^{110}$ Other studies have implicated NK cells as well as NKT cells as significant IL-10 sources during Toxoplasma infection. ${ }^{111,112}$ Clearly, more work is required to resolve these issues.

\section{CONCLUSIONS AND FUTURE DIRECTIONS}

Our knowledge of the mucosal immune responses that are galvanized into action by Toxoplasma and other microbial pathogens has expanded greatly in recent years. Yet, there is still much that remains unknown. We do not fully understand how cells of the mucosal immune system successfully integrate signals received simultaneously from microbial pathogens and commensal microbiota during infection, nor do we understand the breakdown in tolerance that leads to dysbiosis in the intestine. Knowledge gained through use of experimental models such as Toxoplasma will lead to new ways of understanding as well as treating infection and inflammation at the borders of the intestinal mucosa.

\section{DISCLOSURE}

The authors declare no conflict of interest.

(c) 2014 Society for Mucosal Immunology

\section{REFERENCES}

1. Kayama, H. \& Takeda, K. Regulation of intestinal homeostasis by innate and adaptive immunity. Int. Immunol. 24, 673-680 (2012).

2. Nutsch, K.M. \& Hsieh, C.-S. T cell tolerance and immunity to commensal bacteria. Curr. Opin. Immunol. 24, 385-391 (2012).

3. Sansonetti, P.J. To be or not to be a pathogen: that is the mucosally relevant question. Mucosal Immunol. 4, 8-14 (2011).

4. Cader, M.Z. \& Kaser, A. Recent advances in inflammatory bowel disease: mucosal immune cells in intestinal inflammation. Gut 62, 1653-1664 (2013).

5. Kaser, A., Zeissig, S. \& Blumberg, R.S. Inflammatory bowel disease. Annu. Rev. Immunol. 28, 573-621 (2010).

6. Xavier, R.J. \& Podolsky, D.K. Unravelling the pathogenesis of inflammatory bowel disease. Nature 448, 427-434 (2007).

7. McLeod, R., Van Tubbergen, C., Montoya, J.G. \& Petersen, E. In Toxoplasma gondii (Weiss, L.M. \& Kim, K.) 100-162 (Academic Press: Amsterdam, 2014).

8. Dubey, J.P. In Toxoplasma gondii (Weiss, L.M. \& Kim, K.) 1-20 (Academic Press: Amsterdam, 2014).

9. Denkers, E.Y. \& Gazzinelli, R.T. Regulation and function of T-cell-mediated immunity during Toxoplasma gondii infection. Clin. Microbiol. Rev. 11 569-588 (1998).

10. Dupont, C.D., Christian, D.A. \& Hunter, C.A. Immune response and immunopathology during toxoplasmosis. Semin. Immunopathol. 34, 793-813 (2012).
11. Egan, C.E., Cohen, S.B. \& Denkers, E.Y. Insights into inflammatory bowel disease using Toxoplasma gondii as an infectious trigger. Immunol. Cell Biol. 90, 668-675 (2012).

12. Barragan, A., Brossier, F. \& Sibley, L.D. Transepithelial migration of Toxoplasma gondii involves an interaction of intercellular adhesion molecule 1 (ICAM-1) with the parasite adhesin MIC2. Cell Microbiol. 7, 561-568 (2005).

13. Weight, C.M. \& Carding, S.R. The protozoan pathogen Toxoplasma gondii targets the paracellular pathway to invade the intestinal epithelium. Ann. N. Y. Acad. Sci. 1258, 135-142 (2012).

14. Barragan, A. \& Sibley, L.D. Transepithelial migration of Toxoplasma gondii is linked to parasite motility and virulence. J. Exp. Med. 195, 1625-1633 (2002).

15. Barragan, A. \& Sibley, L.D. Migration of Toxoplasma gondii across biological barriers. Trends Microbiol. 11, 426-430 (2003).

16. Gregg, B. et al. Replication and distribution of Toxoplasma gondii in the small intestine after oral infection with tissue cysts. Infect. Immun. 81, 1635-1643 (2013).

17. Coombes, J.L. et al. Motile invaded neutrophils in the small intestine of Toxoplasma gondii-infected mice reveal a potential mechanism for parasite spread. Proc. Natl Acad. Sci. USA 110, E1913-E1922 (2013).

18. Molloy, M.J. et al. Intraluminal containment of commensal outgrowth in the gut during infection-induced dysbiosis. Cell Host Microbe 14, 318-328 (2013).

19. Bierly, A.L., Shufesky, W.J., Sukhumavasi, W., Morelli, A.E. \& Denkers, E.Y. Dendritic cells expressing plasmacytoid marker PDCA-1 are Trojan horses during Toxoplasma gondii infection. J. Immunol. 181, 8485-8491 (2008).

20. Courret, N. et al. CD11c- and CD11b-expressing mouse leukocytes transport single Toxoplasma gondii tachyzoites to the brain. Blood 107, 309-316 (2006).

21. Fuks, J.M. et al. GABAergic signaling is linked to a hypermigratory phenotype in dendritic cells infected by Toxoplasma gondii. PLoS Pathog. 8, e1003051 (2012).

22. Weidner, J.M. \& Barragan, A. Tightly regulated migratory subversion of immune cells promotes the dissemination of Toxoplasma gondii. Int. J. Parasitol. 44, 85-90 (2013).

23. Lambert, H., Hitziger, N., Dellacasa, I., Svensson, M. \& Barragan, A. Induction of dendritic cell migration upon Toxoplasma gondii infection potentiates parasite dissemination. Cell Microbiol. 8, 1611-1623 (2006).

24. Liu, C.-H. et al. Cutting edge: dendritic cells are essential for in vivo IL-12 production and development of resistance against Toxoplasma gondii infection in mice. J. Immunol. 177, 31-35 (2006).

25. Bliss, S.K., Zhang, Y. \& Denkers, E.Y. Murine neutrophil stimulation by Toxoplasma gondii antigen drives high level production of IFN-gammaindependent IL-12. J. Immunol. 163, 2081-2088 (1999).

26. Hou, B., Benson, A., Kuzmich, L., DeFranco, A.L. \& Yarovinsky, F. Critical coordination of innate immune defense against Toxoplasma gondii by dendritic cells responding via their Toll-like receptors. Proc. Natl Acad. Sci. USA 108, 278-283 (2011).

27. Pepper, M. et al. Plasmacytoid dendritic cells are activated by Toxoplasma gondii to present antigen and produce cytokines. J. Immunol. 180, 6229-6236 (2008).

28. Cohen, S.B. et al. CXCR3-dependent $\mathrm{CD}^{+}{ }^{+} \mathrm{T}$ cells are required to activate inflammatory monocytes for defense against intestinal infection. PLoS Pathog. 9, e1003706 (2013).

29. Dunay, I.R., Fuchs, A. \& Sibley, L.D. Inflammatory monocytes but not neutrophils are necessary to control infection with Toxoplasma gondii in mice. Infect. Immun. 78, 1564-1570 (2010).

30. Meredith, M.M. et al. Expression of the zinc finger transcription factor zDC (Zbtb46, Btbd4) defines the classical dendritic cell lineage. J. Exp. Med. 209, 1153-1165 (2012).

31. Dunay, I.R. et al. Gr1(+) inflammatory monocytes are required for mucosal resistance to the pathogen Toxoplasma gondii. Immunity $\mathbf{2 9}$, 306-317 (2008)

32. Edelson, B.T. et al. Peripheral CD103 + dendritic cells form a unified subset developmentally related to CD8alpha + conventional dendritic cells. J. Exp. Med. 207, 823-836 (2010).

33. Jaiswal, H. et al. Batf3 and Id2 have a synergistic effect on Irf8-directed classical CD $8 \alpha+$ dendritic cell development. J. Immunol. 191, 5993-6001 (2013). 
34. Mashayekhi, M. et al. CD8 $\alpha(+)$ dendritic cells are the critical source of interleukin-12 that controls acute infection by Toxoplasma gondii tachyzoites. Immunity 35, 249-259 (2011).

35. Benson, A., Pifer, R., Behrendt, C.L., Hooper, L.V. \& Yarovinsky, F. Gut commensal bacteria direct a protective immune response against Toxoplasma gondii. Cell Host Microbe 6, 187-196 (2009).

36. Mucida, D. et al. Reciprocal TH17 and regulatory T cell differentiation mediated by retinoic acid. Science 317, 256-260 (2007).

37. Coombes, J.L. et al. A functionally specialized population of mucosal CD103 + DCs induces Foxp3 + regulatory T cells via a TGF-beta and retinoic acid-dependent mechanism. J. Exp. Med 204, 1757-1764 (2007).

38. Sun, C.-M. et al. Small intestine lamina propria dendritic cells promote de novo generation of Foxp3 T reg cells via retinoic acid. J. Exp. Med. 204, 1775-1785 (2007).

39. Hall, J.A. et al. Essential role for retinoic acid in the promotion of CD4(+) T cell effector responses via retinoic acid receptor alpha. Immunity 34, 435-447 (2011).

40. Denkers, E.Y. Toll-like receptor initiated host defense against Toxoplasma gondii. J. Biomed. Biotechnol. 2010, 1-8 (2010).

41. Gazzinelli, R.T. \& Denkers, E.Y. Protozoan encounters with Toll-like receptor signalling pathways: implications for host parasitism. Nat. Rev. Immunol. 6, 895-906 (2006).

42. Yarovinsky, F. Toll-like receptors and their role in host resistance to Toxoplasma gondii. Immunol. Lett. 119, 17-21 (2008).

43. Béla, S.R. et al. Impaired innate immunity in mice deficient in interleukin-1 receptor-associated kinase 4 leads to defective type 1 Tcell responses, $\mathrm{B}$ cell expansion, and enhanced susceptibility to infection with Toxoplasma gondii. Infect. Immun. 80, 4298-4308 (2012).

44. Scanga, C.A. et al. Cutting edge: MyD88 is required for resistance to Toxoplasma gondii infection and regulates parasite-induced IL-12 production by dendritic cells. J. Immunol. 168, 5997-6001 (2002).

45. Sukhumavasi, W. et al. TLR adaptor MyD88 is essential for pathogen control during oral Toxoplasma gondii infection but not adaptive immunity induced by a vaccine strain of the parasite. J. Immunol. 181, 3464-3473 (2008).

46. Melo, M.B. et al. UNC93B1 mediates host resistance to infection with Toxoplasma gondii. PLoS Pathog. 6, e1001071 (2010).

47. Pifer, R., Benson, A., Sturge, C.R. \& Yarovinsky, F. UNC93B1 is essential for TLR11 activation and IL-12-dependent host resistance to Toxoplasma gondii. J. Biol. Chem. 286, 3307-3314 (2011).

48. Yarovinsky, F. et al. TLR11 activation of dendritic cells by a protozoan profilin-like protein. Science 308, 1626-1629 (2005).

49. Andrade, W.A. et al. Combined action of nucleic acid-sensing Toll-like receptors and TLR11/TLR12 heterodimers imparts resistance to Toxoplasma gondii in mice. Cell Host Microbe 13, 42-53 (2013).

50. Koblansky, A.A. et al. Recognition of profilin by Toll-like receptor 12 is critical for host resistance to Toxoplasma gondii. Immunity 38, 119-130 (2012).

51. Raetz, M. et al. Cooperation of TLR12 and TLR11 in the IRF8-dependent IL-12 response to Toxoplasma gondii profilin. J. Immunol. 191, 4818-4827 (2013).

52. Plattner, F. et al. Toxoplasma profilin is essential for host cell invasion and TLR11-dependent induction of an interleukin-12 response. Cell Host Microbe 3, 77-87 (2008).

53. Janeway, C.A. Approaching the asymptote? Evolution and revolution in immunology. Cold Spring Harb. Symp. Quant. Biol. 54 (Pt 1), 1-13 (1989).

54. Pifer, R. \& Yarovinsky, F. Innate responses to Toxoplasma gondii in mice and humans. Trends Parasitol. 27, 388-393 (2011).

55. Debierre-Grockiego, F. et al. Activation of TLR2 and TLR4 by glycosylphosphatidylinositols derived from Toxoplasma gondii. J. Immunol. 179, 1129-1137 (2007).

56. Debierre-Grockiego, F., Molitor, N., Schwarz, R.T. \& Lüder, C.G.K Toxoplasma gondii glycosylphosphatidylinositols up-regulate major histocompatibility complex (MHC) molecule expression on primary murine macrophages. Innate Immun. 15, 25-32 (2009).

57. Rosowski, E.E. et al. Strain-specific activation of the NF-kappaB pathway by GRA15, a novel Toxoplasma gondii dense granule protein. J. Exp. Med. 208, 195-212 (2011).

58. Kim, L. et al. Toxoplasma gondii genotype determines MyD88-dependent signaling in infected macrophages. J. Immunol. 177, 2584-2591 (2006).
59. Melo, M.B., Jensen, K.D.C. \& Saeij, J.P.J. Toxoplasma gondii effectors are master regulators of the inflammatory response. Trends Parasitol. 27, 487-495 (2011)

60. Hand, T.W. et al. Acute gastrointestinal infection induces long-lived microbiota-specific T cell responses. Science 337, 1553-1556 (2012).

61. Guiton, R. et al. Interleukin 17 receptor signaling is deleterious during Toxoplasma gondii infection in susceptible BL6 mice. J. Infect. Dis. 202, 427-435 (2010).

62. Kelly, M.N. et al. Interleukin-17/interleukin-17 receptor-mediated signaling is important for generation of an optimal polymorphonuclear response against Toxoplasma gondii infection. Infect. Immun. 73, 617-621 (2005).

63. Muñoz, M. et al. Interleukin (IL)-23 mediates Toxoplasma gondii-induced immunopathology in the gut via matrixmetalloproteinase-2 and IL-22 but independent of IL-17. J. Exp. Med. 206, 3047-3059 (2009).

64. Liesenfeld, O. et al. TNF-alpha, nitric oxide and IFN-gamma are all critical for development of necrosis in the small intestine and early mortality in genetically susceptible mice infected perorally with Toxoplasma gondii. Parasite Immunol. 21, 365-376 (1999).

65. Liesenfeld, O. Oral infection of C57BL/6 mice with Toxoplasma gondii: a new model of inflammatory bowel disease? J. Infect. Dis. 185 (Suppl 1), S96-101 (2002).

66. Craven, M. et al. Inflammation drives dysbiosis and bacterial invasion in murine models of ileal Crohn's disease. PLoS One 7, e41594 (2012).

67. Heimesaat, M.M. et al. Gram-negative bacteria aggravate murine small intestinal Th1-type immunopathology following oral infection with Toxoplasma gondii. J. Immunol. 177, 8785-8795 (2006).

68. Heimesaat, M.M. et al. Exacerbation of murine ileitis by Toll-like receptor 4 mediated sensing of lipopolysaccharide from commensal Escherichia coli. Gut 56, 941-948 (2007).

69. Egan, C.E. et al. Synergy between intraepithelial lymphocytes and lamina propria T cells drives intestinal inflammation during infection. Mucosal Immunol. 4, 658-670 (2011).

70. Clevers, H.C. \& Bevins, C.L. Paneth cells: maestros of the small intestinal crypts. Annu. Rev. Physiol. 75, 289-311 (2013).

71. Foureau, D.M. et al. TLR9-dependent induction of intestinal alphadefensins by Toxoplasma gondii. J. Immunol. 184, 7022-7029 (2010).

72. Raetz, M. et al. Parasite-induced TH1 cells and intestinal dysbiosis cooperate in IFN- $\gamma$-dependent elimination of Paneth cells. Nat. Immunol. 14, 136-142 (2013)

73. Wehkamp, J. et al. Reduced Paneth cell alpha-defensins in ileal Crohn's disease. Proc. Natl Acad. Sci. USA 102, 18129-18134 (2005).

74. Shi, C. \& Pamer, E.G. Monocyte recruitment during infection and inflammation. Nat. Rev. Immunol. 11, 762-774 (2011).

75. Serbina, N.V., Salazar-Mather, T.P., Biron, C.A., Kuziel, W.A. \& Pamer, E.G. TNF/iNOS-producing dendritic cells mediate innate immune defense against bacterial infection. Immunity 19, 59-70 (2003).

76. Serbina, N.V. \& Pamer, E.G. Monocyte emigration from bone marrow during bacterial infection requires signals mediated by chemokine receptor CCR2. Nat. Immunol. 7, 311-317 (2006).

77. Schulthess, J. et al. Interleukin-15-dependent NKp46 + innate lymphoid cells control intestinal inflammation by recruiting inflammatory monocytes. Immunity 37, 108-121 (2012).

78. Khan, I.A. et al. Mice lacking the chemokine receptor CCR1 show increased susceptibility to Toxoplasma gondii infection. J. Immunol. 166. 1930-1937 (2001).

79. Goldszmid, R.S. et al. NK cell-derived interferon- $\gamma$ orchestrates cellular dynamics and the differentiation of monocytes into dendritic cells at the site of infection. Immunity 36, 1047-1059 (2012).

80. Serbina, N.V., Jia, T., Hohl, T.M. \& Pamer, E.G. Monocyte-mediated defense against microbial pathogens. Annu. Rev. Immunol. 26, 421-452 (2008).

81. Scharton-Kersten, T.M., Yap, G. \& Magram, J. Inducible nitric oxide is essential for host control of persistent but not acute infection with the intracellular pathogen Toxoplasma gondii. J. Exp. Med. 185, 1261-1273 (1997).

82. Scharton-Kersten, T.M. et al. In the absence of endogenous IFN-gamma, mice develop unimpaired IL-12 responses to Toxoplasma gondii while failing to control acute infection. J. Immunol. 157, 4045-4054 (1996).

83. Howard, J.C., Hunn, J.P. \& Steinfeldt, T. The IRG protein-based resistance mechanism in mice and its relation to virulence in Toxoplasma gondii. Curr. Opin. Microbiol. 14, 414-421 (2011). 
84. Khaminets, A. et al. Coordinated loading of IRG resistance GTPases on to the Toxoplasma gondii parasitophorous vacuole. Cell Microbiol. 12, 939-961 (2010).

85. Zhao, Y.O., Khaminets, A., Hunn, J.P. \& Howard, J.C. Disruption of the Toxoplasma gondii parasitophorous vacuole by IFNgamma-inducible immunity-related GTPases (IRG proteins) triggers necrotic cell death. PLoS Pathog. 5, e1000288 (2009).

86. Martens, S. et al. Disruption of Toxoplasma gondii parasitophorous vacuoles by the mouse p47-resistance GTPases. PLoS Pathog. 1, e24 (2005).

87. Liesenfeld, O. et al. The IFN- $\gamma$-inducible GTPase, Irga6, protects mice against Toxoplasma gondii but not against Plasmodium berghei and some other intracellular pathogens. PLOS ONE 6, e20568 (2011).

88. Yamamoto, M. etal. A cluster of interferon- $\gamma$-inducible p65 GTPases plays a critical role in host defense against Toxoplasma gondii. Immunity $\mathbf{3 7}$, 302-313 (2012).

89. Grainger, J.R. et al. Inflammatory monocytes regulate pathologic responses to commensals during acute gastrointestinal infection. Nat. Med. 19, 713-721 (2013).

90. Kalinski, P. Regulation of immune responses by prostaglandin E2. J. Immunol. 188, 21-28 (2012).

91. Kunisawa, J., Takahashi, I. \& Kiyono, H. Intraepithelial lymphocytes: their shared and divergent immunological behaviors in the small and large intestine. Immunol. Rev. 215, 136-153 (2007).

92. Cheroutre, H., Lambolez, F. \& Mucida, D. The light and dark sides of intestinal intraepithelial lymphocytes. Nat. Rev. Immunol. 11, 445-456 (2011).

93. Dalton, J.E. et al. Intraepithelial gammadelta + lymphocytes maintain the integrity of intestinal epithelial tight junctions in response to infection. Gastroenterology 131, 818-829 (2006).

94. Chardès, T., Buzoni-Gatel, D., Lepage, A., Bernard, F. \& Bout, D. Toxoplasma gondii oral infection induces specific cytotoxic CD8 alpha/ beta + Thy-1 + gut intraepithelial lymphocytes, lytic for parasite-infected enterocytes. J. Immunol. 153, 4596-4603 (1994).

95. Buzoni-Gatel, D. et al. Murine ileitis after intracellular parasite infection is controlled by TGF-beta-producing intraepithelial lymphocytes. Gastroenterology 120, 914-924 (2001).

96. Buzoni-Gatel, D. et al. Intraepithelial lymphocytes traffic to the intestine and enhance resistance to Toxoplasma gondii oral infection. J. Immunol. 162, 5846-5852 (1999).

97. Luangsay, S. et al. CCR5 mediates specific migration of Toxoplasma gondii-primed CD8 lymphocytes to inflammatory intestinal epithelial cells. Gastroenterology 125, 491-500 (2003).

98. Lepage, A.C., Buzoni-Gatel, D., Bout, D.T. \& Kasper, L.H. Gut-derived intraepithelial lymphocytes induce long term immunity against Toxoplasma gondii. J. Immunol. 161, 4902-4908 (1998).
99. Mennechet, F.J.D. et al. Lamina propria CD4 + T lymphocytes synergize with murine intestinal epithelial cells to enhance proinflammatory response against an intracellular pathogen. J. Immunol. 168, 2988-2996 (2002).

100. Mennechet, F.J.D. et al. Intestinal intraepithelial lymphocytes prevent pathogen-driven inflammation and regulate the Smad/T-bet pathway of lamina propria CD4 + T cells. Eur. J. Immunol. 34, 1059-1067 (2004).

101. Egan, C.E. et al. CCR2-dependent intraepithelial lymphocytes mediate inflammatory gut pathology during Toxoplasma gondii infection. Mucosal Immunol. 2, 527-535 (2009).

102. Liesenfeld, O., Kosek, J., Remington, J.S. \& Suzuki, Y. Association of CD4 + T cell-dependent, interferon-gamma-mediated necrosis of the small intestine with genetic susceptibility of mice to peroral infection with Toxoplasma gondii. J. Exp. Med. 184, 597-607 (1996).

103. Hall, A.O. et al. The cytokines interleukin 27 and interferon- $\gamma$ promote distinct Treg cell populations required to limit infection-induced pathology. Immunity 37, 511-523 (2012).

104. Bollrath, J. \& Powrie, F.M. Controlling the frontier: regulatory T-cells and intestinal homeostasis. Semin. Immunol. 25, 352-357 (2013).

105. Gazzinelli, R.T. et al. In the absence of endogenous IL-10, mice acutely infected with Toxoplasma gondii succumb to a lethal immune response dependent on CD4 + T cells and accompanied by overproduction of IL-12, IFN-gamma and TNF-alpha. J. Immunol. 157, 798-805 (1996).

106. Suzuki, Y. et al. IL-10 is required for prevention of necrosis in the small intestine and mortality in both genetically resistant BALB/C and susceptible C57BL/6 mice following peroral infection with Toxoplasma gondii. J. Immunol. 164, 5375-5382 (2000).

107. Oldenhove, G. et al. Decrease of Foxp3 + Treg cell number and acquisition of effector cell phenotype during lethal infection. Immunity $\mathbf{3 1}$, 772-786 (2009).

108. Benson, A. et al. Microbial infection-induced expansion of effector T cells overcomes the suppressive effects of regulatory $\mathrm{T}$ cells via an $\mathrm{IL}-2$ deprivation mechanism. J. Immunol. 188, 800-810 (2012).

109. Roers, A. et al. Tcell-specific inactivation of the interleukin 10 gene in mice results in enhanced $T$ cell responses but normal innate responses to lipopolysaccharide or skin irritation. J. Exp. Med. 200, 1289-1297 (2004).

110. Jankovic, D. et al. Conventional T-bet(+)Foxp3(-) Th1 cells are the major source of host-protective regulatory $\mathrm{IL}-10$ during intracellular protozoan infection. J. Exp. Med. 204, 273-283 (2007).

111. Ronet, C. et al. NKT cells are critical for the initiation of an inflammatory bowel response against Toxoplasma gondii. J. Immunol. 175, 899-908 (2005).

112. Perona-Wright, G. et al. Systemic but not local infections elicit immunosuppressive IL-10 production by natural killer cells. Cell Host Microbe 6, 503-512 (2009). 